\title{
New Hermite-Hadamard type inequalities for product of different convex functions involving certain fractional integral operators
}

\author{
Erhan Set ${ }^{\mathrm{a}}$, Junesang Choi ${ }^{\mathrm{b}, *}$, Barış Çelik ${ }^{\mathrm{a}}$ \\ ${ }^{a}$ Department of Mathematics, Faculty of Science and Arts, Ordu University, Ordu, Turkey. \\ ${ }^{b}$ Department of Mathematics, Dongguk University, Gyeongju 38066, Republic of Korea.
}

\begin{abstract}
We aim to establish new Hermite-Hadamard type inequalities for products of two different convex functions involving certain generalized fractional integral operators. The results presented here, being very general, are pointed out to be specialized to yield many new and known inequalities associated with some known fractional integral operators.
\end{abstract}

Keywords: Convex function, s-convex function, Hermite-Hadamard type inequalities, fractional integral operators.

2010 MSC: 26A33, 26D10, 26D15, 33B15.

(C)2018 All rights reserved.

\section{Introduction and preliminaries}

Here we recall some definitions and some known results for easier reference and later use. Let I be an interval in $\mathbb{R}$. A function $f: I \rightarrow \mathbb{R}$ is said to be convex if the inequality

$$
f(t x+(1-t) y) \leqslant t f(x)+(1-t) f(y),
$$

holds for all $x, y \in I$ and $t \in[0,1]$. Here and in the following, let $\mathbb{C}, \mathbb{R}, \mathbb{R}^{+}, \mathbb{N}$ and $\mathbb{Z}_{0}^{-}$be the sets of complex numbers, real numbers, positive real numbers, positive integers and non-positive integers, respectively, and let $\mathbb{R}_{0}^{+}:=\mathbb{R}^{+} \cup\{0\}$ and $\mathbb{N}_{0}:=\mathbb{N} \cup\{0\}$. For this convex function $f: I \rightarrow \mathbb{R}$, the following well-known inequality is referred to, in the literature, as the Hermite-Hadamard integral inequality:

$$
f\left(\frac{a+b}{2}\right) \leqslant \frac{1}{b-a} \int_{a}^{b} f(x) d x \leqslant \frac{f(a)+f(b)}{2}
$$

where $a, b \in$ I with $a<b$.

\footnotetext{
*Corresponding author

Email addresses: erhanset@yahoo.com (Erhan Set), junesang@mail.dongguk.ac.kr (Junesang Choi),

bariscelik15@hotmail.com (Barış Çelik)

doi: 10.22436/jmcs.018.01.04
}

Received 2017-08-08 
A function $f:[0, \infty) \rightarrow \mathbb{R}$ is said to be s-convex in the second sense if

$$
f(t x+(1-t) y) \leqslant t^{s} f(x)+(1-t)^{s} f(y)
$$

for all $x, y \in[0, \infty), t \in[0,1]$ and for some fixed $s \in(0,1]$.

Breckner [3] introduced the s-convex function in the second sense and Hudzik and Maligranda [9] presented a number of properties and connections with s-convexity in the first sense.

Since fractional calculus was introduced at the end of the nineteenth century, the subject has become a rapidly growing area and has found many applications in various research fields. Due mainly to the demonstrated applications, many researchers have investigated certain inequalities for different classes of functions involving diverse fractional integral operators (see, e.g., $[2,6-8,10,12-16,18]$ ).

We denote by $L_{p}(a, b)(1 \leqslant p \leqslant \infty)$ the set of those Lebesgue complex-valued measurable functions $f$ on $[a, b](-\infty \leqslant a<b \leqslant \infty)$ for which $\|f\|_{p}<\infty$, where

$$
\|f\|_{p}=\left(\int_{a}^{b}|f(t)|^{p} d t\right)^{\frac{1}{p}}, \quad(1 \leqslant p<\infty),
$$

and

$$
\|f\|_{\infty}=\operatorname{ess} \sup _{a \leqslant t \leqslant b}|f(t)| .
$$

Let $f \in L_{1}[a, b]:=L(a, b)$. The Riemann-Liouville integrals $J_{a+}^{\alpha} f$ and $J_{b-}^{\alpha} f$ of order $\alpha \in \mathbb{R}^{+}$with $a \in \mathbb{R}_{0}^{+}$are defined, respectively, by

$$
J_{a+}^{\alpha} f(x)=\frac{1}{\Gamma(\alpha)} \int_{a}^{x}(x-t)^{\alpha-1} f(t) d t, \quad(x>a)
$$

and

$$
J_{b-}^{\alpha} f(x)=\frac{1}{\Gamma(\alpha)} \int_{x}^{b}(t-x)^{\alpha-1} f(t) d t, \quad(x<b),
$$

where $\Gamma$ is the familiar Gamma function (see, e.g., [19, Section 1.1]). It is noted that $J_{a+f}^{1} f(x)$ and $J_{b-}^{1} f(x)$ become the usual Riemann integrals.

The beta function $B(\alpha, \beta)$ is defined by (see, e.g., [19, Section 1.1])

$$
\mathrm{B}(\alpha, \beta)= \begin{cases}\int_{0}^{1} \mathrm{t}^{\alpha-1}(1-\mathrm{t})^{\beta-1} \mathrm{dt}, & (\mathfrak{R}(\alpha)>0 ; \mathfrak{R}(\beta)>0), \\ \frac{\Gamma(\alpha) \Gamma(\beta)}{\Gamma(\alpha+\beta)}, & \left(\alpha, \beta \in \mathbb{C} \backslash \mathbb{Z}_{0}^{-}\right) .\end{cases}
$$

Chen [4] presented Hermite-Hadamard type inequalities for products of two functions, which are recalled in Theorems 1.1 and 1.2.

Theorem 1.1. Let $\mathrm{f}$ and $\mathrm{g}$ be real-valued, nonnegative and convex functions on $[\mathrm{a}, \mathrm{b}]$. Then

$$
\begin{aligned}
& \frac{\Gamma(\alpha+1)}{2(b-a)^{\alpha}}\left[J_{a^{+}}^{\alpha} f(b) g(b)+J_{b^{-}}^{\alpha} f(a) g(a)\right] \\
& \quad \leqslant\left(\frac{\alpha}{\alpha+2}-\frac{\alpha}{\alpha+1}+\frac{1}{2}\right) M(a, b)+\frac{\alpha}{(\alpha+1)(\alpha+2)} N(a, b),
\end{aligned}
$$

where $\alpha \in \mathbb{R}^{+}$,

$$
M(a, b):=f(a) g(a)+f(b) g(b) \text {, and } N(a, b):=f(a) g(b)+f(b) g(a) \text {. }
$$


Theorem 1.2. Let $\mathrm{f}$ and $\mathrm{g}$ be real-valued, nonnegative and convex functions on $[\mathrm{a}, \mathrm{b}]$. Then

$$
\begin{aligned}
2 f\left(\frac{a+b}{2}\right) g\left(\frac{a+b}{2}\right) \leqslant & \frac{\Gamma(\alpha+1)}{2(b-a)^{\alpha}}\left[J_{a^{+}}^{\alpha} f(b) g(b)+J_{b^{-}}^{\alpha} f(a) g(a)\right] \\
& +M(a, b) \frac{\alpha}{(\alpha+1)(\alpha+2)}+N(a, b)\left(\frac{\alpha}{\alpha+2}-\frac{\alpha}{\alpha+1}+\frac{1}{2}\right)
\end{aligned}
$$

where $\alpha \in \mathbb{R}^{+}, M(a, b)$ and $N(a, b)$ are the same as given in (1.3).

Chen and $\mathrm{Wu}[5]$ established Hermite-Hadamard type inequalities for products of two different convex functions, which are recalled in Theorems 1.3, 1.4 and 1.5.

Theorem 1.3. Let $\mathrm{f}, \mathrm{g}:[\mathrm{a}, \mathrm{b}] \rightarrow \mathbb{R}(\mathrm{a}<\mathrm{b})$ with $\mathrm{a}, \mathrm{b} \in[0, \infty)$ be functions such that $\mathrm{f}, \mathrm{g} \in \mathrm{L}[\mathrm{a}, \mathrm{b}]$. Also, let $\mathrm{f}$ be convex and nonnegative and $\mathrm{g}$ be s-convex on $[\mathrm{a}, \mathrm{b}]$ for some fixed $\mathrm{s} \in(0,1]$. Then

$$
\begin{aligned}
\frac{\Gamma(\alpha)}{(b-a)^{\alpha}}\left[J_{a^{+}}^{\alpha} f(b) g(b)+J_{b^{-}}^{\alpha} f(a) g(a)\right] \leqslant & \left(\frac{1}{\alpha+s+1}+B(\alpha, s+2)\right) M(a, b) \\
& +\left(B(\alpha+1, s+1)+\frac{1}{(\alpha+s)(\alpha+s+1)}\right) N(a, b),
\end{aligned}
$$

where $\alpha \in \mathbb{R}^{+}, M(a, b)$ and $N(a, b)$ are the same as given in (1.3).

Theorem 1.4. Let $\mathrm{f}, \mathrm{g}:[\mathrm{a}, \mathrm{b}] \rightarrow \mathbb{R}(\mathrm{a}<\mathrm{b})$ with $\mathrm{a}, \mathrm{b} \in[0, \infty)$ be functions such that $\mathrm{f}, \mathrm{g} \in \mathrm{L}[\mathrm{a}, \mathrm{b}]$. Also, let $\mathrm{f}$ be $s_{1}$-convex and $g$ be $s_{2}$-convex on $[\mathrm{a}, \mathrm{b}]$ for some fixed $\mathrm{s}_{1}, \mathrm{~s}_{2} \in(0,1]$. Then

$$
\begin{aligned}
\frac{\Gamma(\alpha)}{(b-a)^{\alpha}}\left[J_{a^{+}}^{\alpha} f(b) g(b)+J_{b^{-}}^{\alpha} f(a) g(a)\right] \leqslant & \left\{\frac{1}{\alpha+s_{1}+s_{2}}+B\left(\alpha, s_{1}+s_{2}+1\right)\right\} M(a, b) \\
& +\left\{B\left(\alpha+s_{1}, s_{2}+1\right)+B\left(\alpha+s_{2}, s_{1}+1\right)\right\} N(a, b),
\end{aligned}
$$

where $\alpha \in \mathbb{R}^{+}, M(a, b)$ and $N(a, b)$ are the same as given in (1.3).

Theorem 1.5. Let $\mathrm{f}, \mathrm{g}:[\mathrm{a}, \mathrm{b}] \rightarrow \mathbb{R}(\mathrm{a}<\mathrm{b})$ with $\mathrm{a}, \mathrm{b} \in[0, \infty)$ be functions such that $\mathrm{f}, \mathrm{g} \in \mathrm{L}[\mathrm{a}, \mathrm{b}]$. Also, let $\mathrm{f}$ be convex and nonnegative and $\mathrm{g}$ be s-convex on $[\mathrm{a}, \mathrm{b}]$ for some fixed $\mathrm{s} \in(0,1]$. Then

$$
\begin{aligned}
2^{s} f\left(\frac{a+b}{2}\right) g\left(\frac{a+b}{2}\right) \leqslant & \frac{\Gamma(\alpha+1)}{2(b-a)^{\alpha}}\left[J_{a^{+}}^{\alpha} f(b) g(b)+J_{b^{-}}^{\alpha} f(a) g(a)\right] \\
& +\frac{1}{2} M(a, b)\left\{B(\alpha+1, s+1)+\frac{1}{(\alpha+s)(\alpha+s+1)}\right\} \\
& +\frac{1}{2} N(a, b)\left\{B(\alpha, s+2)+\frac{1}{\alpha+s+1}\right\}
\end{aligned}
$$

where $\alpha \in \mathbb{R}^{+}, M(a, b)$ and $N(a, b)$ are the same as given in (1.3).

Raina [11] introduced a class of functions defined formally by

$$
\mathcal{F}_{\rho, \lambda}^{\sigma}(x)=\mathcal{F}_{\rho, \lambda}^{\sigma(0), \sigma(1), \cdots}(x)=\sum_{k=0}^{\infty} \frac{\sigma(k)}{\Gamma(\rho k+\lambda)} x^{k}, \quad\left(\rho, \lambda \in \mathbb{R}^{+} ;|x|<\infty\right),
$$

where the coefficients $\sigma(k) \in \mathbb{R}^{+}\left(k \in \mathbb{N}_{0}\right)$ form a bounded sequence. With the help of (1.8), Raina [11] and Agarwal et al. [1] defined the following left-sided and right-sided fractional integral operators, respectively, as follows:

$$
\left(\mathcal{J}_{\rho, \lambda, a+; w}^{\sigma} \varphi\right)(x)=\int_{a}^{x}(x-t)^{\lambda-1} \mathcal{F}_{\rho, \lambda}^{\sigma}\left[w(x-t)^{\rho}\right] \varphi(t) d t, \quad(a<x),
$$


and

$$
\left(\mathcal{J}_{\rho, \lambda, \mathrm{b}-; w}^{\sigma} \varphi\right)(x)=\int_{x}^{b}(t-x)^{\lambda-1} \mathcal{F}_{\rho, \lambda}^{\sigma}\left[w(t-x)^{\rho}\right] \varphi(t) d t, \quad(x<b),
$$

where $\rho, \lambda \in \mathbb{R}^{+}, w \in \mathbb{R}$ and $\varphi(t)$ is a function such that the integrals on the right sides exist.

It is easy to verify that $\mathcal{J}_{\rho, \lambda, a+; w}^{\sigma} \varphi(x)$ and $\mathcal{J}_{\rho, \lambda, b-; w}^{\sigma} \varphi(x)$ are bounded integral operators on $L(a, b)$ if

$$
\mathfrak{M}:=\mathcal{F}_{\rho, \lambda+1}^{\sigma}\left[w(b-a)^{\rho}\right]<\infty .
$$

In fact, for $\varphi \in \mathrm{L}(\mathrm{a}, \mathrm{b})$, we have

$$
\left\|\partial_{\rho, \lambda, a+; w}^{\sigma} \varphi(x)\right\|_{1} \leqslant \mathfrak{M}(b-a)^{\lambda}\|\varphi\|_{1}
$$

and

$$
\left\|\partial_{\rho, \lambda, b-; w}^{\sigma} \varphi(x)\right\|_{1} \leqslant \mathfrak{M}(b-a)^{\lambda}\|\varphi\|_{1} .
$$

It is noted that many useful fractional integral operators can be obtained by specializing the coefficient $\sigma(k)$ in (1.9) and (1.10), for example, the Riemann-Liouville fractional integrals $J_{a+}^{\alpha}$ and $J_{b-}^{\alpha}$ of order $\alpha$ follow easily by setting $\lambda=\alpha, \sigma(0)=1$ and $w=0$ in (1.9) and (1.10). For some recent results and properties concerning the fractional integral operators (1.9) and (1.10), one may be referred to [17, 20, 21$]$.

Here, we aim to establish certain new Hermite-Hadamard type inequalities for products of two different convex functions involving the fractional integral operators (1.9) and (1.10). The results presented here, being very general, are pointed out to be specialized to yield many new and known inequalities associated with some known fractional integral operators.

\section{Inequalities for product of convex and s-convex functions}

Here we begin by stating a generalized Hermite-Hadamard type inequality for product of a convex function and a s-convex function involving the fractional integral operators (1.9) and (1.10), which is asserted by Theorem 2.1.

Theorem 2.1. Let $\mathrm{f}, \mathrm{g}:[\mathrm{a}, \mathrm{b}] \rightarrow \mathbb{R}_{0}^{+}(\mathrm{a}<\mathrm{b})$ with $\mathrm{a}, \mathrm{b} \in[0, \infty)$ be functions such that $\mathrm{f}, \mathrm{g} \in \mathrm{L}[\mathrm{a}, \mathrm{b}]$. Also, let $\mathrm{f}$ be convex and $\mathrm{g}$ be s-convex on $[\mathrm{a}, \mathrm{b}]$ for some fixed $\mathrm{s} \in(0,1]$. Then

$$
\begin{aligned}
& \frac{1}{(b-a)^{\alpha}}\left[\left(\mathcal{J}_{\rho, \alpha, a+; w}^{\sigma}\right)(f g(b))+\left(\mathcal{J}_{\rho, \alpha, b-; w}^{\sigma}\right)(f g(a))\right] \\
& \leqslant M(a, b) \mathcal{F}_{\rho, \alpha}^{\sigma_{1}}\left[w(b-a)^{\rho}\right]+N(a, b) \mathcal{F}_{\rho, \alpha}^{\sigma_{2}}\left[w(b-a)^{\rho}\right],
\end{aligned}
$$

where $\alpha, \rho, w \in \mathbb{R}^{+}, M(a, b)$ and $\mathrm{N}(\mathrm{a}, \mathrm{b})$ are the same as given in (1.3),

$$
\sigma_{1}(k):=\sigma(k)\left(\frac{1}{\alpha+\rho k+s+1}+B(\alpha+\rho k, s+2)\right)
$$

and

$$
\sigma_{2}(k):=\sigma(k)\left(B(\alpha+\rho k+1, s+1)+\frac{1}{(\alpha+\rho k+s)(\alpha+\rho k+s+1)}\right) .
$$

Proof. We find from the definitions of $f$ and $g$ that, for $t \in[0,1]$,

$$
f(t a+(1-t) b) \leqslant t f(a)+(1-t) f(b),
$$

and

$$
g(t a+(1-t) b) \leqslant t^{s} g(a)+(1-t)^{s} g(b) .
$$


By multiplying side by side of (2.2) and (2.3) and considering that each term is non-negative real, we get for $t \in[0,1]$,

$$
\begin{aligned}
f(t a+(1-t) b) g(t a+(1-t) b) \leqslant & t^{s+1} f(a) g(a)+(1-t)^{s+1} f(b) g(b) \\
& +t(1-t)^{s} f(a) g(b)+(1-t) t^{s} f(b) g(a) .
\end{aligned}
$$

Similarly,

$$
\begin{aligned}
f((1-t) a+t b) g((1-t) a+t b) \leqslant & (1-t)^{s+1} f(a) g(a)+t^{s+1} f(b) g(b) \\
& +(1-t) t^{s} f(a) g(b)+t(1-t)^{s} f(b) g(a) .
\end{aligned}
$$

By adding side by side of (2.4) and (2.5), we obtain

$$
\begin{aligned}
& f(t a+(1-t) b) g(t a+(1-t) b)+f((1-t) a+t b) g((1-t) a+t b) \\
& \leqslant\left\{t^{s+1}+(1-t)^{s+1}\right\} M(a, b)+\left\{t(1-t)^{s}+(1-t) t^{s}\right\} N(a, b),
\end{aligned}
$$

where $M(a, b)$ and $N(a, b)$ are the same as given in (1.3).

Multiplying both sides of $(2.6)$ by $t^{\alpha-1} \mathcal{F}_{\rho, \alpha}^{\sigma}\left[w(b-a)^{\rho} t^{\rho}\right]$ and integrating the resulting inequality with respect to $t$ over $[0,1]$, we obtain

$$
\mathcal{L}_{1}(\alpha, \sigma, \rho, w)+\mathcal{L}_{2}(\alpha, \sigma, \rho, w) \leqslant \mathcal{R}_{1}(\alpha, \sigma, \rho, w, s)+\mathcal{R}_{2}(\alpha, \sigma, \rho, w, s),
$$

where

$$
\begin{aligned}
& \mathcal{L}_{1}(\alpha, \sigma, \rho, w):=\int_{0}^{1} t^{\alpha-1} \mathcal{F}_{\rho, \alpha}^{\sigma}\left[w(b-a)^{\rho} t^{\rho}\right] f(t a+(1-t) b) g(t a+(1-t) b) d t \\
& \mathcal{L}_{2}(\alpha, \sigma, \rho, w):=\int_{0}^{1} t^{\alpha-1} \mathcal{F}_{\rho, \alpha}^{\sigma}\left[w(b-a)^{\rho} t^{\rho}\right] f((1-t) a+t b) g((1-t) a+t b) d t \\
& \mathcal{R}_{1}(\alpha, \sigma, \rho, w, s):=M(a, b) \int_{0}^{1} t^{\alpha-1} \mathcal{F}_{\rho, \alpha}^{\sigma}\left[w(b-a)^{\rho} t^{\rho}\right]\left\{t^{s+1}+(1-t)^{s+1}\right\} d t \\
& \mathcal{R}_{2}(\alpha, \sigma, \rho, w, s):=N(a, b) \int_{0}^{1} t^{\alpha-1} \mathcal{F}_{\rho, \alpha}^{\sigma}\left[w(b-a)^{\rho} t^{\rho}\right]\left\{t(1-t)^{s}+(1-t) t^{s}\right\} d t
\end{aligned}
$$

Setting $t a+(1-t) b=u$ and $(1-t) a+t b=v$ in $\mathcal{L}_{1}(\alpha, \sigma, \rho, w)$ and $\mathcal{L}_{2}(\alpha, \sigma, \rho, w)$, respectively, and using (1.9) and (1.10), we get

$$
\begin{aligned}
\mathcal{L}_{1}(\alpha, \sigma, \rho, w)+\mathcal{L}_{2}(\alpha, \sigma, \rho, w)= & \frac{1}{(b-a)^{\alpha}} \int_{a}^{b}(b-u)^{\alpha-1} \mathcal{F}_{\rho, \alpha}^{\sigma}\left[w(b-u)^{\rho}\right] f(u) g(u) d u \\
& +\frac{1}{(b-a)^{\alpha}} \int_{a}^{b}(v-a)^{\alpha-1} \mathcal{F}_{\rho, \alpha}^{\sigma}\left[w(v-a)^{\rho}\right] f(v) g(v) d v \\
= & \frac{1}{(b-a)^{\alpha}}\left\{\left(\partial_{\rho, \alpha, a+; w}^{\sigma}\right)(f g(b))+\left(\mathcal{g}_{\rho, \alpha, b-; w}^{\sigma}\right)(f g(a))\right\} .
\end{aligned}
$$

Using (1.8), we have

$$
\mathcal{R}_{1}(\alpha, \sigma, \rho, w, s)=M(a, b) \sum_{k=0}^{\infty} \frac{\sigma(k) w^{k}(b-a)^{\rho k}}{\Gamma(\alpha+\rho k)} \int_{0}^{1} t^{\alpha+\rho k-1}\left\{t^{s+1}+(1-t)^{s+1}\right\} d t .
$$

We find from (1.1) that

$$
\begin{aligned}
\int_{0}^{1} t^{\alpha+\rho k-1}\left\{t^{s+1}+(1-t)^{s+1}\right\} d t & =\frac{1}{\alpha+\rho k+s+1}+\int_{0}^{1} t^{\alpha+\rho k-1}(1-t)^{s+1} d t \\
& =\frac{1}{\alpha+\rho k+s+1}+B(\alpha+\rho k, s+2) .
\end{aligned}
$$


In view of (1.8), we thus have

$$
\mathcal{R}_{1}(\alpha, \sigma, \rho, w, s)=M(a, b) \mathcal{F}_{\rho, \alpha}^{\sigma_{1}}\left[w(b-a)^{\rho}\right] .
$$

Similarly, we get

$$
\mathcal{R}_{2}(\alpha, \sigma, \rho, w, s)=\mathrm{N}(\mathrm{a}, \mathrm{b}) \mathcal{F}_{\rho, \alpha}^{\sigma_{2}}\left[w(b-a)^{\rho}\right] .
$$

Finally, substituting (2.8), (2.9), and (2.10) in (2.7), we are led to the desired result (2.1).

Remark 2.2. Setting $\sigma(0)=1$ and $w=0$ in Theorem 2.1, we find that the inequality (2.1) reduces to the inequality (1.5).

Theorem 2.3. Let $\mathrm{f}, \mathrm{g}:[\mathrm{a}, \mathrm{b}] \rightarrow \mathbb{R}_{0}^{+}(\mathrm{a}<\mathrm{b})$ with $\mathrm{a}, \mathrm{b} \in[0, \infty)$ be functions such that $\mathrm{f}, \mathrm{g}, \mathrm{fg} \in \mathrm{L}[\mathrm{a}, \mathrm{b}]$. Also, let $f$ and $g$ be $s_{1}$-convex and $s_{2}$-convex on $[\mathrm{a}, \mathrm{b}]$ for some fixed $\mathrm{s}_{1}, \mathrm{~s}_{2} \in(0,1]$, respectively. Then

$$
\begin{aligned}
\frac{1}{(b-a)^{\alpha}} & \left\{\left(\mathcal{J}_{\rho, \alpha, a+; w}^{\sigma}\right)(f g(b))+\left(\mathcal{J}_{\rho, \alpha, b-; w}^{\sigma}\right)(f g(a))\right\} \\
& \leqslant M(a, b) \mathcal{F}_{\rho, \alpha}^{\sigma_{3}}\left[w(b-a)^{\rho}\right]+N(a, b) \mathcal{F}_{\rho, \alpha}^{\sigma_{4}}\left[w(b-a)^{\rho}\right],
\end{aligned}
$$

where $\alpha, \rho, w \in \mathbb{R}^{+}, M(a, b)$ and $N(a, b)$ are the same as given in (1.3),

$$
\begin{aligned}
& \sigma_{3}(k):=\sigma(k)\left\{\frac{1}{\alpha+\rho k+s_{1}+s_{2}}+B\left(\alpha+\rho k, s_{1}+s_{2}+1\right)\right\}, \\
& \sigma_{4}(k):=\sigma(k)\left\{B\left(\alpha+\rho k+s_{1}, s_{2}+1\right)+B\left(\alpha+\rho k+s_{2}, s_{1}+1\right)\right\} .
\end{aligned}
$$

Proof. A similar argument as in the proof of Theorem 2.1 will establish the result here. We omit the details.

Corollary 2.4. Under the assumptions of Theorem 2.3 with $s_{1}=s_{2}=1$, we have

$$
\begin{aligned}
\frac{1}{(b-a)^{\alpha}} & \left\{\left(\mathcal{J}_{\rho, \alpha, a+; w}^{\sigma}\right)(f g(b))+\left(\mathcal{J}_{\rho, \alpha, b-; w}^{\sigma}\right)(f g(a))\right\} \\
& \leqslant M(a, b) \mathcal{F}_{\rho, \alpha}^{\sigma_{5}}\left[w(b-a)^{\rho}\right]+N(a, b) \mathcal{F}_{\rho, \alpha}^{\sigma_{6}}\left[w(b-a)^{\rho}\right],
\end{aligned}
$$

where $\alpha, \rho, w \in \mathbb{R}^{+}, M(a, b)$ and $\mathrm{N}(\mathrm{a}, \mathrm{b})$ are the same as given in (1.3),

$$
\begin{aligned}
& \sigma_{5}(k):=\sigma(k)\left(\frac{2}{\alpha+\rho k+2}-\frac{2}{\alpha+\rho k+1}+\frac{1}{\alpha+\rho k}\right), \\
& \sigma_{6}(k):=\frac{2 \sigma(k)}{(\alpha+\rho k+1)(\alpha+\rho k+2)} .
\end{aligned}
$$

Remark 2.5. Setting $\sigma(0)=1$ and $w=0$ in Theorem 2.3, we find that the inequality (2.11) reduces to the inequality (1.6). Taking $\sigma(0)=1$ and $w=0$ in Corollary 2.4, we see that the inequality (2.12) reduces to the inequality (1.2).

Theorem 2.6. Let $\mathrm{f}, \mathrm{g}:[\mathrm{a}, \mathrm{b}] \rightarrow \mathbb{R}_{0}^{+}(\mathrm{a}<\mathrm{b})$ with $\mathrm{a}, \mathrm{b} \in[0, \infty)$ be functions such that $\mathrm{fg} \in \mathrm{L}[\mathrm{a}, \mathrm{b}]$. Also, let $\mathrm{f}$ be convex and $\mathrm{g}$ be s-convex on $[\mathrm{a}, \mathrm{b}]$ for some fixed $\mathrm{s} \in(0,1]$. Then

$$
\begin{aligned}
2^{s+1} f\left(\frac{a+b}{2}\right) g\left(\frac{a+b}{2}\right) \mathcal{F}_{\rho, \alpha}^{\sigma_{7}}\left[w(b-a)^{\rho}\right] \leqslant & \frac{1}{(b-a)^{\alpha}}\left\{\left(\mathcal{J}_{\rho, \alpha, a+; w}^{\sigma}\right)(f g(b))+\left(\mathcal{J}_{\rho, \alpha, b-; w}^{\sigma}\right)(f g(a))\right\} \\
& +M(a, b) \mathcal{F}_{\rho, \alpha}^{\sigma_{2}}\left[w(b-a)^{\rho}\right]+N(a, b) \mathcal{F}_{\rho, \alpha}^{\sigma_{1}}\left[w(b-a)^{\rho}\right],
\end{aligned}
$$

where $\alpha, \rho, w \in \mathbb{R}^{+}, M(a, b)$ and $N(a, b)$ are the same as given in (1.3), $\sigma_{1}$ and $\sigma_{2}$ are the same as given in Theorem 2.1,

$$
\sigma_{7}(k):=\frac{\sigma(k)}{\alpha+\rho k}
$$


Proof. By using the definitions of $f$ and $g$, we have (see $[5, \mathrm{p} .714]$ )

$$
\begin{aligned}
f\left(\frac{a+b}{2}\right) g\left(\frac{a+b}{2}\right)= & f\left(\frac{t a+(1-t) b}{2}+\frac{(1-t) a+t b}{2}\right) g\left(\frac{t a+(1-t) b}{2}+\frac{(1-t) a+t b}{2}\right) \\
\leqslant & \frac{1}{2^{s+1}}[f(t a+(1-t) b) g(t a+(1-t) b) \\
& +f((1-t) a+t b) g((1-t) a+t b)] \\
& +\frac{1}{2^{s+1}}\left[\left\{t(1-t)^{s}+(1-t) t^{s}\right\} M(a, b)\right. \\
& \left.+\left\{(1-t)^{s+1}+t^{s+1}\right\} N(a, b)\right] .
\end{aligned}
$$

Now, applying a similar argument to (2.14) as in the proof of Theorem 2.1 will establish the result here. We omit the details.

Corollary 2.7. Under the assumptions of Theorem 2.6 with $\mathrm{s}=1$, we have

$$
\begin{aligned}
4 f\left(\frac{a+b}{2}\right) g\left(\frac{a+b}{2}\right) \mathcal{F}_{\rho, \alpha}^{\sigma_{7}}\left[w(b-a)^{\rho}\right] \leqslant & \frac{1}{(b-a)^{\alpha}}\left\{\left(\partial_{\rho, \alpha, a+; w}^{\sigma}\right)(f g(b))+\left(\mathcal{J}_{\rho, \alpha, b-; w}^{\sigma}\right)(f g(a))\right\} \\
& +M(a, b) \mathcal{F}_{\rho, \alpha}^{\sigma_{6}}\left[w(b-a)^{\rho}\right]+N(a, b) \mathcal{F}_{\rho, \alpha}^{\sigma_{5}}\left[w(b-a)^{\rho}\right],
\end{aligned}
$$

where $\alpha, \rho, w \in \mathbb{R}^{+}, M(a, b)$ and $N(a, b)$ are the same as given in (1.3), $\sigma_{5}$ and $\sigma_{6}$ are the same as in Corollary 2.4, and $\sigma_{7}$ is the same as given in Theorem 2.6.

Remark 2.8. Setting $\sigma(0)=1$ and $w=0$ in Theorem 2.6, we find that the inequality (2.13) reduces to the inequality (1.7). Taking $\sigma(0)=1$ and $w=0$ in Corollary 2.7, we see that the inequality (2.15) reduces to the inequality (1.4).

\section{Acknowledgment}

This research is supported by Ordu University Scientific Research Projects Coordination Unit (BAP). Project Number: BY-1716.

\section{References}

[1] R. P. Agarwal, M.-J. Luo, R. K. Raina, On Ostrowski type inequalities, Fasc. Math., 56 (2016), 5-27. 1

[2] M. U. Awan, M. A. Noor, M. V. Mihai, K. I. Noor, Fractional Hermite-Hadamard inequalities for differentiablesGodunova-Levin functions, Filomat, 30 (2016), 3235-3241. 1

[3] W. W. Breckner, Stetigkeitsaussagen fr eine Klasse verallgemeinerter konvexer funktionen in topologischen linearen Raumen, Pupl. Inst. Math., 23 (1978), 13-20. 1

[4] F. Chen, A note on Hermite-Hadamard inequalities for products of convex functions via Riemann-Liouville fractional integrals, Ital. J. Pure Appl. Math., 33 (2014), 299-306. 1

[5] F. Chen, S. Wu, Several complementary inequalities to inequalities of Hermite-Hadamard type for s-convex functions, J. Nonlinear Sci. Appl., 9 (2016), 705-716. 1, 2

[6] Z. Dahmani, New inequalities in fractional integrals, Int. J. Nonlinear Sci., 9 (2010), 493-497. 1

[7] Z. Dahmani, L. Tabharit, S. Taf, Some fractional integral inequalities, Nonlinear. Sci. Lett. A, 2 (2010), 155-160.

[8] R. Gorenflo, F. Mainardi, Fractional Calculus: Integral and Differential Equations of Fractional Order, Springer Verlag, Vienna, (1997). 1

[9] H. Hudzik, L. Maligranda, Some remarks on s-convex functions, Aequationes Math., 48 (1994), 100-111. 1

[10] M. A. Noor, K. I. Noor, M. U. Awan, Fractional Hermite-Hadamard inequalities for two kinds of s-preinvex functions, Nonlinear Sci. Lett. A, 8 (2017), 11-24. 1

[11] R. K. Raina, On generalized Wright's hypergeometric functions and fractional calculus operators, East Asian Math. J., 21 (2005), 191-203. 1, 1

[12] S. G. Samko, A. A. Kilbas, O. I. Marichev, Fractional Integrals and Derivatives (Theory and Applications), Gordon and Breach, Switzerland, (1993). 1

[13] M. Z. Sarıkaya, E. Set, H. Yaldız, N. Başak, Hermite-Hadamards inequalities for fractional integrals and related fractional inequalities, Math. Comput. Model., 57 (2013), 2403-2407. 
[14] E. Set, A. O. Akdemir, B. Çelik, Some Hermite-Hadamard type inequalities for products of two different convex functions via conformable fractional integrals, Xth International Statistics Days Conference, Turkey, (2016).

[15] E. Set, B. Çelik, Certain Hermite-Hadamard type inequalities associated with conformable fractional integral operators, Foundations, Submitted.

[16] E. Set, J. Choi, B. Çelik, Certain Hermite-Hadamard type inequalities involving generalized fractional integral operators, RACSAM, 2017 (2017), 9 pages. 1

[17] E. Set, A. Gözpınar, Some new inequalities involving generalized fractional integral operators for several class functions, AIP Conference Proceedings, (2017). 1

[18] E. Set, M. Z. Sarıkaya, M. E. Özdemir, H. Yıldırım, The Hermite-Hadamard's inequality for some convex functions via fractional integrals and related results, J. Appl. Math. Stat. Inform., 10 (2014), 69-83. 1

[19] H. M. Srivastava, J. Choi, Zeta and q-Zeta Functions and Associated Series and Integrals, Elsevier, Amsterdam, (2012). 1

[20] F. Usta, H. Budak, M. Z. Sarıkaya, E. Set, On generalization of trapezoid type inequalities for s-convex functions with generalized fractional integral operators, https://www.researchgate.net/publication/312596720. 1

[21] H. Yaldı, M. Z. Sarikaya, On the Hermite-Hadamard type inequalities for fractional integral operator, https://www . researchgate.net/publication/309824275. 1 